\title{
Propagation of a reaction front in a narrow sample of energetic material with heat losses: chaotic regimes, extinction and intermittency
}

\author{
Vadim N. Kurdyumov ${ }^{a, 1}$, Vladimir V. Gubernov ${ }^{b, c}$ \\ ${ }^{a}$ Department of Energy, CIEMAT, Avda. Complutense 22, 28040 Madrid, Spain \\ ${ }^{b}$ P.N. Lebedev Physical Institute, 53 Leninsky pr., Moscow 119991, Russia \\ ${ }^{c}$ Far Easten Federal University, 8 Suhanova st., Vladivostok 690950, Russia
}

\begin{abstract}
The influence of heat-losses on the flame dynamics in narrow samples of energetic material is investigated numerically. The model is reduced to a one-dimensional form with the flame-sheet approximation applied for the reaction rate. Both the steady-state solutions and its linear stability analysis are treated analytically. A typical C-shaped response curve is found for the dependence of the flame-propagation velocity on the heat-loss parameter, with solutions along the lower branch of slower flames being always unstable. It is found that a part of the upper branch of the C-shaped response curve is also unstable and the PoincaréAndronov-Hopf bifurcation takes place at a certain value of heat-loss intensity even if the steady state solution is stable under the corresponding adiabatic conditions.

The numerical simulations show that an increase in heat-losses induces, for sufficiently high Zeldovich numbers, the Feigenbaum's cascade of period doubling bifurcations after which a chaotic dynamics is setting in. The chaotic dynamics precedes the flame extinction occurring for the further increase of the heat-loss parameter which, nevertheless, remains significantly lower than the steady extinction limit dictated by the C-shaped response curve. Apparently, the parametric dependence of the extinction time in these cases is also irregular with appreciable disparities in magnitude. Finally, the intermittency effect is detected slightly below the extinction limit with irregular dynamics alternating by apparently periodic stages. These results may be important for the flammability limits theory and practical fire safety applications.
\end{abstract}

\section{Introduction}

The emergence of oscillatory (diffusive-thermal) instabilities due to the Poincaré-AndronovHopf bifurcation (PAH below) is well known in combustion of solid fuels. One-dimensional pul-

\footnotetext{
${ }^{1}$ Corresponding author.
} 
sations in the propagation of combustion waves were found in experiments on high-temperature self propagating synthesis, combustion of thermites and gunpowders [1-3]. The onset of multidimensional traveling instabilities may result in the formation of complex unsteady dynamical structures such as spinning waves [4-9]. It was shown that an increase in heat-losses promotes the flame instability for values of the Zel'dovich number lower than under the corresponding adiabatic conditions [9].

It was established that as the parameters of the system are modified away from the neutral stability boundary for the onset of pulsations the complex regimes of flame oscillations may develop. The period doubling bifurcations were found in experiments on the burning of tantalumcarbon mixtures [3]. These dynamical regimes were investigated numerically first in [10] within the one-step model with the Arrhenius dependence of the reaction rate on the temperature.

It is well known that the onset of oscillations may lead to quenching. For laminar flames it was found in experiments with burner-stabilized flames [11] that as the flammability limit is approached the dynamics of combustion resembles chaotic and, then, at certain stage the flame is extinguished. It is remarkable that flame can extinguish long before the steady extinction limit is reached in the parametric space [12]. A hypothesis was proposed that the extinction occurs due to the crisis of chaotic attractor. This proposition was corroborated in numerical analysis of the oscillatory dynamics of combustion waves in the model with chain-branching reactions [12]. Besides that for detonation waves [13] it was found that the development of pulsations may lead to extinction without the chaotic regime route as the amplitude of oscillations grows beyond a certain threshold value. The nature of dynamical quenching is still unclear and needs further analysis.

The analytical study of complex unsteady one-dimensional pulsating regimes of combustion wave propagation was carried out in [14] by means of a weak nonlinear analysis. Two models for the propagation of adiabatic combustion waves were considered in [15]. One of the models included melting of components of the solid fuel, while the other did not. In both cases the increase of the activation energy of the reaction causes flame pulsations via the PAH bifurcation. In the model without melting further increase of the bifurcation parameter results in the subsequent emergence of the solutions of period two, four and eight. It was assumed (however it was not proved rigorously) that the chaotic regime of flame oscillations appears as a result of infinite sequence of period doubling bifurcations. In contrast to that in the model with melting the solutions of period two were only detected. The solutions of period one and two sequentially emerged and disappeared as the bifurcation parameter was increased. The model with melting 
was investigated also in [16] with attention on temporally resonant mode interactions.

In a number of papers [17-19] the free boundary models are employed in order to investigate the diffusive-thermal stability of solid fuel combustion. Two scenarios for the onset of pulsations were suggested depending on the choice of kinetic functions used in the models. The traveling combustion waves lose stability either via the PAH bifurcation followed by the Feigenbaum's cascade of period doubling bifurcations or the stability is lost via the infinite period bifurcation of the Shilnikov type. Both super- and subcritical PAH bifurcations as well as multiple cascades of period doubling were found in [19].

It should be noted that in the majority of the above studies the influence of heat-losses on the flame dynamics was not investigated. The effect of heat losses on the pulsating behavior of combustion waves in solid fuels was studied in [20]. It was shown that a sequence of period doubling bifurcations emerges leading to the formation of solutions of period two and four. The possibility of the existence of the infinite cascade of period doubling bifurcations leading to chaos was discussed. However, the numerical calculations presented in [20] showed that the increase of bifurcation parameter results in the flame quenching without emergence of solutions with period higher than four. Thus the mechanism of the dynamical flame quenching, emergence of chaotic solutions and the role of chaos in the extinction of combustion waves are still unclear even for the one-dimensional one-step models of flame propagation in solid fuels.

It must be admitted that the flame-sheet model, when considering unsteady propagation, is a phenomenological one and can not be derived asymptotically in a rigorous way. In fact, it is a result of truncation of the asymptotic series expansion [21]. It is not a case for non-adiabatic flames with Lewis number close to unity investigated in the pioneering work [22] where linear stability analysis of a planar flame with weak heat losses was carried out. Nevertheless, the flame-sheet model is very often used in modeling of flame propagation in both solids and gas mixtures elucidating many aspects of flame dynamics. A detailed discussion on the closure problem and ways to circumvent it can be found in [23], where, particularly, the effect of incomplete fuel combustion was taken into account.

It has been recently demonstrated in [24] that the inclusion of highly heat conducting element into the solid fuel samples allows the effective control of the dynamics of pulsating combustion waves including the chaotic regimes. The direct and reverse cascades of period doubling bifurcations can be initiated by the proper choice of parameters.

In this work we consider the model where the reaction chemistry is single step reaction with rate proportional to the concentration of fuel and Arrhenius type temperature dependence. Such 
approach is valid, for example, for mono-propellants or mixtures with a single limiting component of the combustion reaction. We assume that the reaction is gasless and there is no filtration processes involved as it occurs in the case of combustion of thermites. The model considers mixture as marcoscopic homogeneous continuous media and all microscopic effects, e.g. granular structure of the mixture, local melting/recrystallization, melt flow dynamics and capillary effects etc., are neglected. All physical properties of the solid media are constant and do not depend on temperature and conversion of fresh mixture into products. Such approach has been successfully employed in many papers on solid phase combustion to describe qualitatively the flame speed, structure, stability and dynamics. In the current work we are focused on the specific case of one dimensional pulsating dynamics of deflagration waves in solid fuel combustion. The purpose of this work is to study how the heat-losses affect the mechanism of transition to chaos and quenching of combustion waves in such systems.

\section{The mathematical formulation}

Consider a deflagration process in a narrow sample of energetic material at initial temperature $T_{0}$. Combustion is modeled by an irreversible reaction of the form $F \rightarrow P+Q$, where $F$ denotes the combustible substance, $P$ is the product, and $Q$ is the heat released per unit mass of fuel. In the present study we define the characteristic length and time using relations $\delta_{T}=\alpha / S_{L}$ and $t_{c}=\alpha / S_{L}^{2}$, where $S_{L}$ is the planar flame speed and $\alpha$ is the (constant) thermal diffusivity; $\delta_{T}$ denotes the thermal flame thickness. The mass fraction is normalized below with respect to its upstream value, $Y_{0}$, and the non-dimensional temperature, $\theta=\left(T-T_{0}\right) /\left(T_{a}-T_{0}\right)$, is based on the adiabatic flame temperature $T_{a}=T_{0}+Q Y_{0} / c$ being $c$ the (constant) specific heat capacity.

The non-dimensional governing equations, written in the reference frame attached to the flame front, and the boundary conditions upstream and downstream, take the form

$$
\begin{gathered}
\frac{\partial \theta}{\partial t}+u_{f} \frac{\partial \theta}{\partial x}=\frac{\partial^{2} \theta}{\partial x^{2}}+\omega-k \theta, \\
\frac{\partial Y}{\partial t}+u_{f} \frac{\partial Y}{\partial x}=-\omega, \\
x \rightarrow-\infty: \quad \theta=Y-1=0 ; \quad x \rightarrow \infty: \quad \partial^{2} \theta / \partial x^{2}=0,
\end{gathered}
$$

where $u_{f}$ is the flame velocity measured with $S_{L}$. The fuel mass fraction equation contains only the first spatial derivative and no outlet boundary condition is required. This mild downstream 
temperature condition replaces a more severe zero-temperature condition which should be imposed at $x \rightarrow \infty$ when the heat losses are not negligible. Notice that it is valid also for the adiabatic case $k=0$. The numerical simulations reported below showed that the influence of the downstream boundary condition becomes negligible, as it should be, if the size of a computational domain is reasonably large.

In the present study the well-known flame-sheet model, which can be traced back to [25], is applied. It consists in approximation of the chemical reaction by a concentrated heat source at the front

$$
\omega=F\left(\theta_{f}\right) \delta(x), \quad \text { with } \quad F\left(\theta_{f}\right)=\exp \left\{\frac{\beta}{2} \frac{\theta_{f}-1}{\left[1+\gamma\left(\theta_{f}-1\right)\right]}\right\},
$$

where $\theta_{f} \equiv \theta(0, t)$ is the non-dimensional flame temperature and $\delta(x)$ is the Dirac deltafunction.

It is remarkable that the above formulation has only three parameters : $\beta=E\left(T_{a}-T_{0}\right) / \mathcal{R} T_{a}^{2}$, the Zeldovich number, where $E$ is the activation energy and $\mathcal{R}$ is the universal gas constant, $\gamma=\left(T_{a}-T_{0}\right) / T_{a}$, the heat release parameter, and $k$ which measures the heat-loss intensity. In all calculations presented below $\gamma=0.7$ was fixed as a representative value. The influence of $\gamma$ on the results will be reported elsewhere.

Assuming outside the flame sheet $Y=1$ for $x<0$ and $Y=0$ for $x>0$, integration of the mass fraction equation (2) around $x=0$ provides

$$
u_{f}=F\left(\theta_{f}\right)=\exp \left\{\frac{\beta}{2} \frac{\theta_{f}-1}{\left[1+\gamma\left(\theta_{f}-1\right)\right]}\right\} .
$$

It should be stressed that complete consumption of the reactant has been assumed in Eq. (5), as differentiated from that in [23] where fuel leakage was allowed. Thus, the flame dynamics is described by the only equation

$$
\frac{\partial \theta}{\partial t}+F\left(\theta_{f}\right) \frac{\partial \theta}{\partial x}=\frac{\partial^{2} \theta}{\partial x^{2}}-k \theta
$$

to be solved separately in each of the regions $x<0$ and $x>0$, with the boundary conditions (3), subject to the following jump conditions at the flame front $x=0$,

$$
[\theta]=0, \quad\left[\frac{\partial \theta}{\partial x}\right]+F\left(\theta_{f}\right)=0, \quad \text { where } \quad \theta_{f}=\theta(0, t) .
$$

Here $[f] \equiv f\left(0^{+}\right)-f\left(0^{-}\right)$denotes the jump of quantity $f$ across the front. For the adiabatic case, $k=0$, the steady-state solution of Eqs. (6)-(7) reads

$$
\theta_{a 0}(x)= \begin{cases}e^{x}, & x<0 \\ 1, & x>0\end{cases}
$$


providing $\theta_{f}=1$ and $u_{f}=1$. It is worth noting that the above formulation is completely identical to that explored in $[14,17]$ with the only exception of the heat-loss term added to the right-hand side of Eq. (6). Anticipating the results presented below the influence of this term on the flame dynamics becomes crucial.

\section{The basic solution and its stability}

In what follows the steady-state variables are denoted by subindex "0". It is well known that the heat-losses can cause multiplicity of solutions when for the same set of parameters more than one steady-states exist. It is convenient to use the steady-state flame velocity, $u_{f 0}$, as a parameter to describe the steady-state. Let us introduce the following quantities

$$
a_{1}=\frac{u_{f 0}-\sqrt{u_{f 0}^{2}+4 k}}{2}, \quad a_{2}=\frac{u_{f 0}+\sqrt{u_{f 0}^{2}+4 k}}{2} .
$$

Using the first jump condition (7), the steady-state solution of Eqs. (6) takes the form

$$
\theta_{0}= \begin{cases}\theta_{f 0} \exp \left(a_{1} x\right), & x>0 \\ \theta_{f 0} \exp \left(a_{2} x\right), & x<0\end{cases}
$$

where

$$
\theta_{f 0}=\frac{\beta-(2 \gamma-2) \ln u_{f 0}}{\beta-2 \gamma \ln u_{f 0}}
$$

follows from Eq. (5). Substituting Eq. (10) into the second jump condition (7) leads to

$$
k=\frac{\left[(2 \gamma-1) \ln u_{f 0}-\beta\right]}{\left[2(\gamma-1) \ln u_{f 0}-\beta\right]^{2}} u_{f 0}^{2} \ln u_{f 0} .
$$

This dependence is plotted in Fig. 1 for $\gamma=0.7$ and various $\beta$ showing a typical C-shaped response curve.

The above steady-state solution is perturbed now as usual with small perturbations

$$
\theta=\theta_{0}(x)+\epsilon e^{\lambda t} \Phi(x)
$$

where $\lambda$ is a complex number the real part of which gives the growth rate and $\epsilon \ll 1$ is the perturbation amplitude. The linearized eigenvalue problem obtained when substituting Eq. (12) into Eqs. (6)-(7) reduces to finding the non-trivial solutions of the following system

$$
\lambda \Phi+u_{f 0} \Phi^{\prime}+u_{f 1} \Phi(0) \theta_{0}^{\prime}=\Phi^{\prime \prime}-k \Phi,
$$




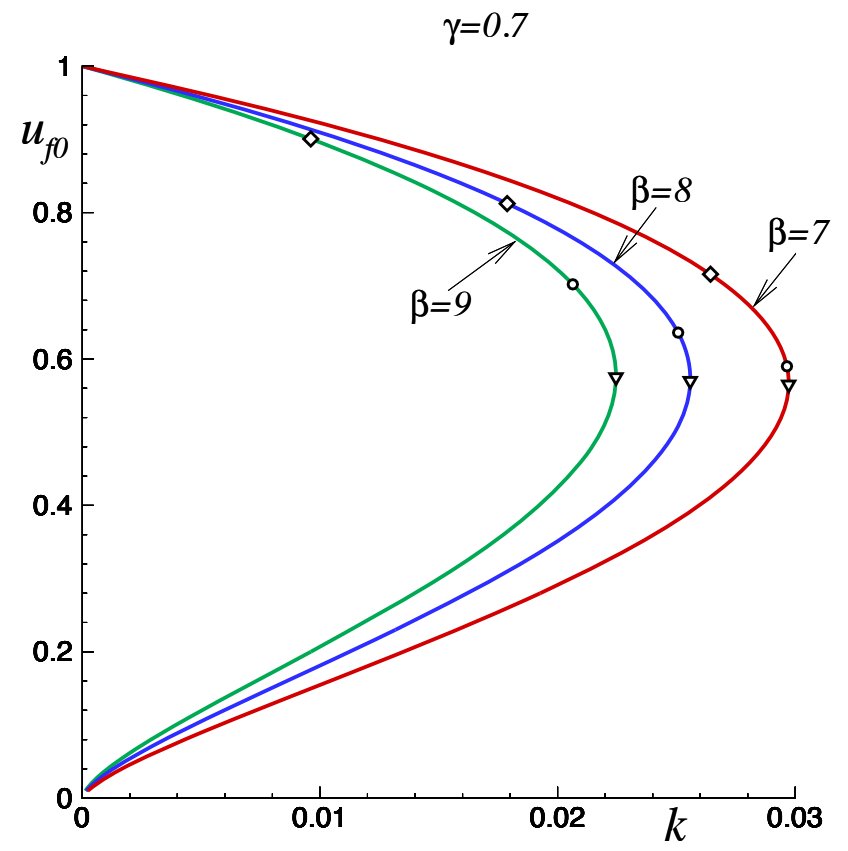

Figure 1: Steady state flame velocity $u_{f}$ as a function of $k$ plotted for various $\beta$; gradient symbols indicate the turning points; open circles - the points where the complex conjugate eigenvalues becomes real; diamond symbols - the extinction values.

$$
[\Phi]=0, \quad\left[\Phi^{\prime}\right]+u_{f 1} \Phi(0)=0, \quad x \rightarrow \pm \infty: \quad|\Phi|=0,
$$

where

$$
u_{f 1}=d F\left(\theta_{f 0}\right) / d \theta_{f 0}=\frac{\beta}{2\left[1+\gamma\left(\theta_{f 0}-1\right)\right]^{2}} \exp \left\{\frac{\beta}{2} \frac{\theta_{f 0}-1}{\left[1+\gamma\left(\theta_{f 0}-1\right)\right]}\right\}
$$

In the following the eigenfunction is normalized by $\Phi(0)=1$ without loss of generality.

To determine whether a basic state is unstable we seek conditions under which $\operatorname{Re}(\lambda)$ is nonnegative. The general solution of Eqs. (13)-(14) takes the form

$$
\Phi(x)= \begin{cases}-\lambda^{-1} u_{f 1} \theta_{f 0} a_{1} e^{a_{1} x}+c_{1} e^{\mu_{1} x}, & x>0 ; \\ -\lambda^{-1} u_{f 1} \theta_{f 0} a_{2} e^{a_{2} x}+c_{2} e^{\mu_{2} x}, & x<0\end{cases}
$$

where

$$
\mu_{1}=\frac{u_{f 0}-\sqrt{u_{f 0}^{2}+4(\lambda+k)}}{2}, \quad \mu_{2}=\frac{u_{f 0}+\sqrt{u_{f 0}^{2}+4(\lambda+k)}}{2}
$$



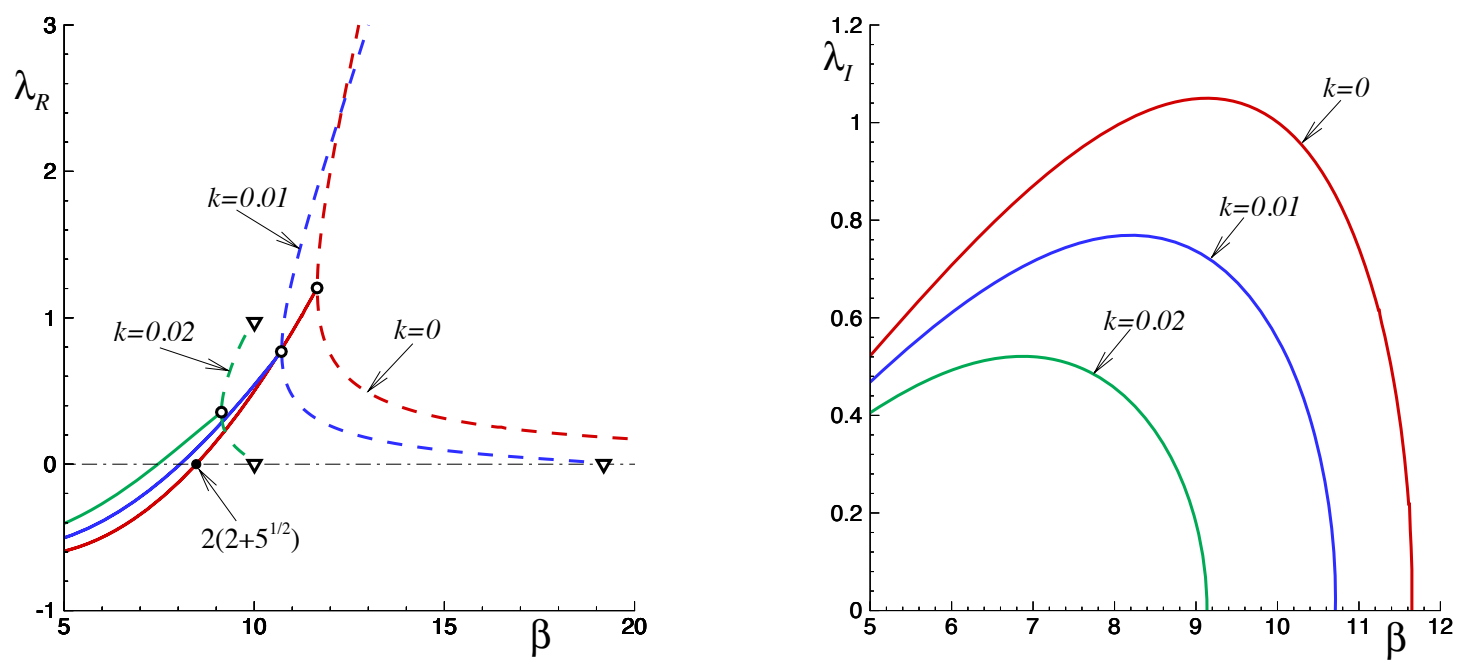

Figure 2: The growth rate $\lambda_{R}$ (left) and the frequency of oscillations $\lambda_{I}$ (right) as a function of $\beta$ calculated for $k=0,0.01$ and $k=0.02$ along the upper branches of the response curves shown in Fig. 1; $\gamma=0.7$ for all curves. In the left figure solid lines show complex conjugated eigenvalues and dashed lines show real eigenvalues; gradient symbols correspond to the turning points shown with the same symbols in Fig. 1.

The above solution is valid for $\lambda_{R}>-k-\lambda_{I}^{2} / u_{f 0}^{2}$ where $R e\left(\mu_{1}\right)<0$. Substituting Eq. (15) into the jump conditions (14) provides the solvability condition to find the eigenvalues

$$
\left(u_{f 0} u_{f 1} \theta_{f 0}+2 \lambda\right) \sqrt{u_{f 0}^{2}+4 \lambda+4 k}-u_{f 0} u_{f 1} \theta_{f 0} \sqrt{u_{f 0}^{2}+4 k}-2 \lambda u_{f 1}=0
$$

Although this leads to an analytical expression for roots, writing it down has been not provided due to its long length. The real and imaginary parts of $\lambda$ are plotted as functions of $\beta$ for $k=0$, 0.01 and 0.02 in Fig. 2 evaluated along the upper branch of the C-shaped curve $u_{f 0}$ versus $k$. The solid lines represent to the complex eigenvalues and the dashed lines show the real ones. The adiabatic case, $k=0$ (for which $u_{f 1}=\beta / 2$ ), was investigated in [14] where the critical Zeldovich number $\beta_{c}=2(2+\sqrt{5}) \approx 8.472$ (re-written in terms of the present variables) above which the steady-state solution is unstable was found. It is indicated with a dark circle in Fig. 2 (left). Notice, that for a fixed non-zero value of $k$ the steady solution exists only up to a finite value of $\beta$ corresponding to the turning points indicated with gradient symbols in Fig. 1. These points are also indicated with gradient symbols in Fig. 2 (left). 

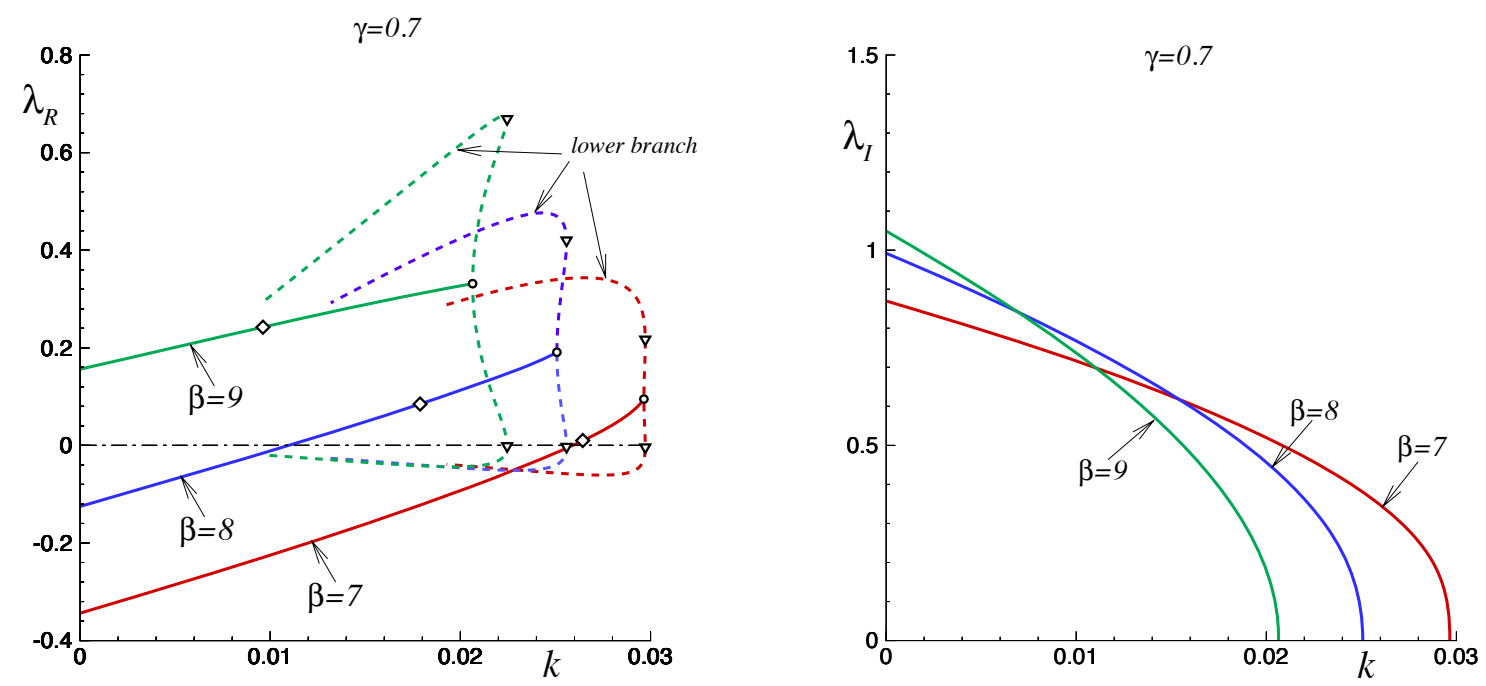

Figure 3: The growth rate $\lambda_{R}$ (left) and the frequency of oscillations $\lambda_{I}$ (right) as a function of $k$ calculated for $\beta=7,8$ and 9 ; all curves for $\gamma=0.7$. In the left figure solid lines show complex conjugated eigenvalues and dashed lines show real eigenvalues; gradient symbols correspond to the turning points shown with the same symbols in Fig. 1; diamond symbols indicate extinction values.

The real and imaginary parts of $\lambda$ evaluated along both the upper and lower branches of the response curve are plotted in Fig. 3 as functions of $k$ for three values of $\beta$ and $\gamma=0.7$. The solid lines correspond to the complex eigenvalues and the dashed lines are used to plot the real eigenvalues.

The steady solutions corresponding to the lower branch of the response curve are always unconditionally unstable with one positive (above the upper gradient symbol) and other negative (below the lower gradient symbol) eigenvalues. One can see that along the upper branch increasing heat-losses promote instability of the traveling wave solution which becomes unstable above a critical value even if the steady-state is stable for $k=0$, as shown in the cases with $\beta=7$ and 8. Open circles show the points where two complex conjugate eigenvalues merge becoming real. Gradient symbols indicate the turning points of the response curves plotted in Fig. 1.

The stability results are summarized in Fig. 4 showing the curves $k$ versus $\beta$ plotted for $\gamma=0.7$. The steady-state solutions exist below the dash-dotted line corresponding to the turning points (gradient symbols) in Fig. 1. Below the solid line indicating the PAH bifurcation the 


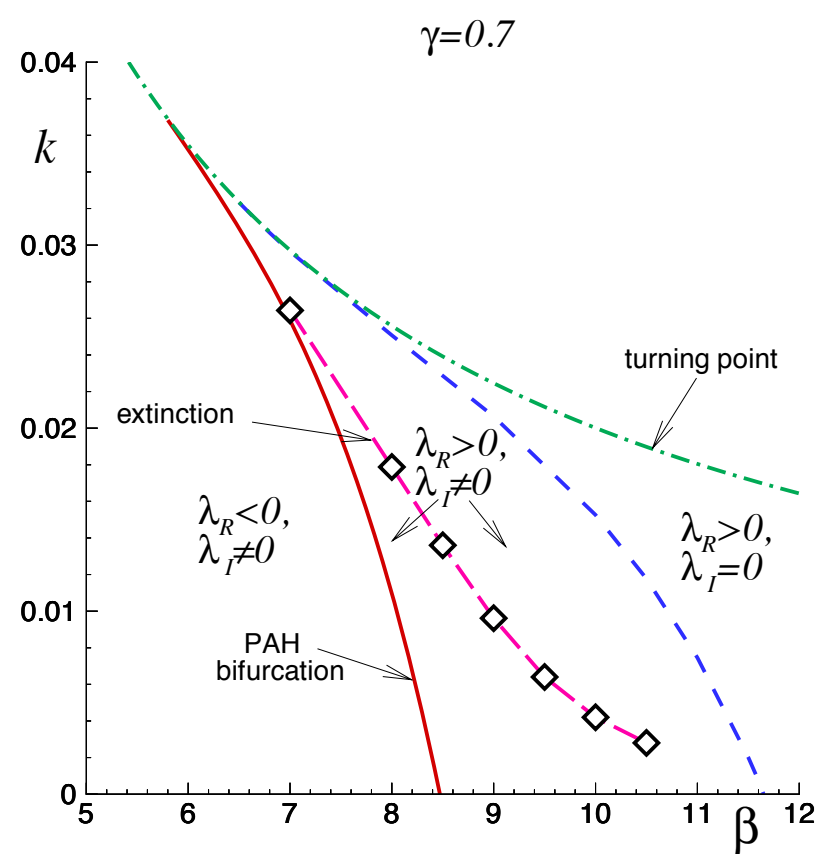

Figure 4: The stability map $k$ versus $\beta$ plotted for $\gamma=0.7$; the steady-state solutions exist below the dash-dotted line; the solid line shows the PAH bifurcation; above the dashed line the eigenvalues are real; the dashed line with diamonds shows the extinction values.

upper branch steady solutions are stable. Between the solid and dashed lines the eigenvalues are complex while above the dashed line they are real. Anticipating the results of numerical simulations, we show in Fig. 4 with a long-dash line and diamond symbols the extinction values above which the flame does not survive. These values are also shown with diamond symbols in Figs. 1 and 3 (left).

\section{Numerical results}

\subsection{Numerical treatment}

Time-dependent simulations of Eq. (6) were carried out in a finite domain, $x_{\min }<x<x_{\max }$ with the typical values $x_{\min }=-20$ and $x_{\max }=20$. The spatial derivatives were disretized on a uniform grid using second order three-point central differences and an explicit, first order, time 
marching procedure was used with the typical time step $\tau=2 \cdot 10^{-5}$. The typical number of grid points was 2001. The number of grid points was doubled in some cases and $\tau$ was halved without any significant differences in the results. The domain size was also extended to $x_{\min }=-60$ and $x_{\max }=60$. The explicit in time procedure allowed to write the jump condition for the temperature derivatives given by Eq. (14) in the form of a non-linear equation for $\theta_{f}$ further resolved iteratively at every time step with accuracy $10^{-15}$. It should be noted that in the frame of the flame sheet model the size the reaction zone is infinitely small and, thus, the requirement for the mesh size requiring a certain number of grid points inside the reaction region becomes of no importance.

The initial condition for time dependent simulations was used in the form

$$
t=0: \quad \theta=\theta_{a 0}(x)+\theta_{h} e^{-x^{2}},
$$

where $\theta_{a 0}(x)$ is given by Eq. (8). In the following, the amplitude of perturbation $\theta_{h}$ was zero unless otherwise specified.

\subsection{Adiabatic case}

Consider first the adiabatic case imposing $k=0$ in Eq. (6). Figure 5 shows the time-histories of $u_{f}$ calculated for various values of $\beta$ below and above the critical value $\beta_{c}=8.4721$ obtained from the linear stability analysis. The case $\beta=8$ was calculated using $\theta_{h}=0.1$ in the initial condition (17). One can see that for $\beta<\beta_{c}$ the initial perturbations decay in accordance with the stability curves plotted in Fig. 2. For $\beta>\beta_{c}$ the time-periodic behavior is formed and the unique period doubling bifurcation occurs at $\beta \approx 9.47$. For further increasing $\beta$ no more period doubling bifurcations were observed. The relaxation dynamics comes into particular prominence for large $\beta$ illustrated with the case $\beta=12$ where very sharp peaks of the flame velocity are alternated by extensive intervals with quasi-steady very slow motion. Figure 2 shows that in the adiabatic case, $k=0$, the eigenvalues become real for $\beta>\beta_{*}=11.65$ marked here with an open circle. It should be particularly emphasized that no chaotic behavior was observed for $k=0$ up to $\beta=12$. The result obtained for $k=0$ indicates the lack of the Feigenbaum's cascade of period doubling bifurcations, at least for $\gamma=0.7$ fixed in the present study. The similar results were reported in [17]. 



Figure 5: Time histories of the flame velocity calculated for the adiabatic sample $(k=0)$ for $\gamma=0.7$ and several $\beta ; \theta_{h}=0.1$ was used for $\beta=8$.

\subsection{Non-adiabatic samples: chaotic behavior and extinction}

Figure 6 shows the time-histories of $u_{f}$ calculated for $\beta=7$ and various $k$. The corresponding critical heat-loss parameter obtained from the linear stability analysis above which the flame becomes unstable is $k_{c} \approx 0.02589$. Numerical simulations show the flame dynamics in accordance with the linear stability analysis. For values of $k=0.025<k_{c}$ the flame approaches after a 

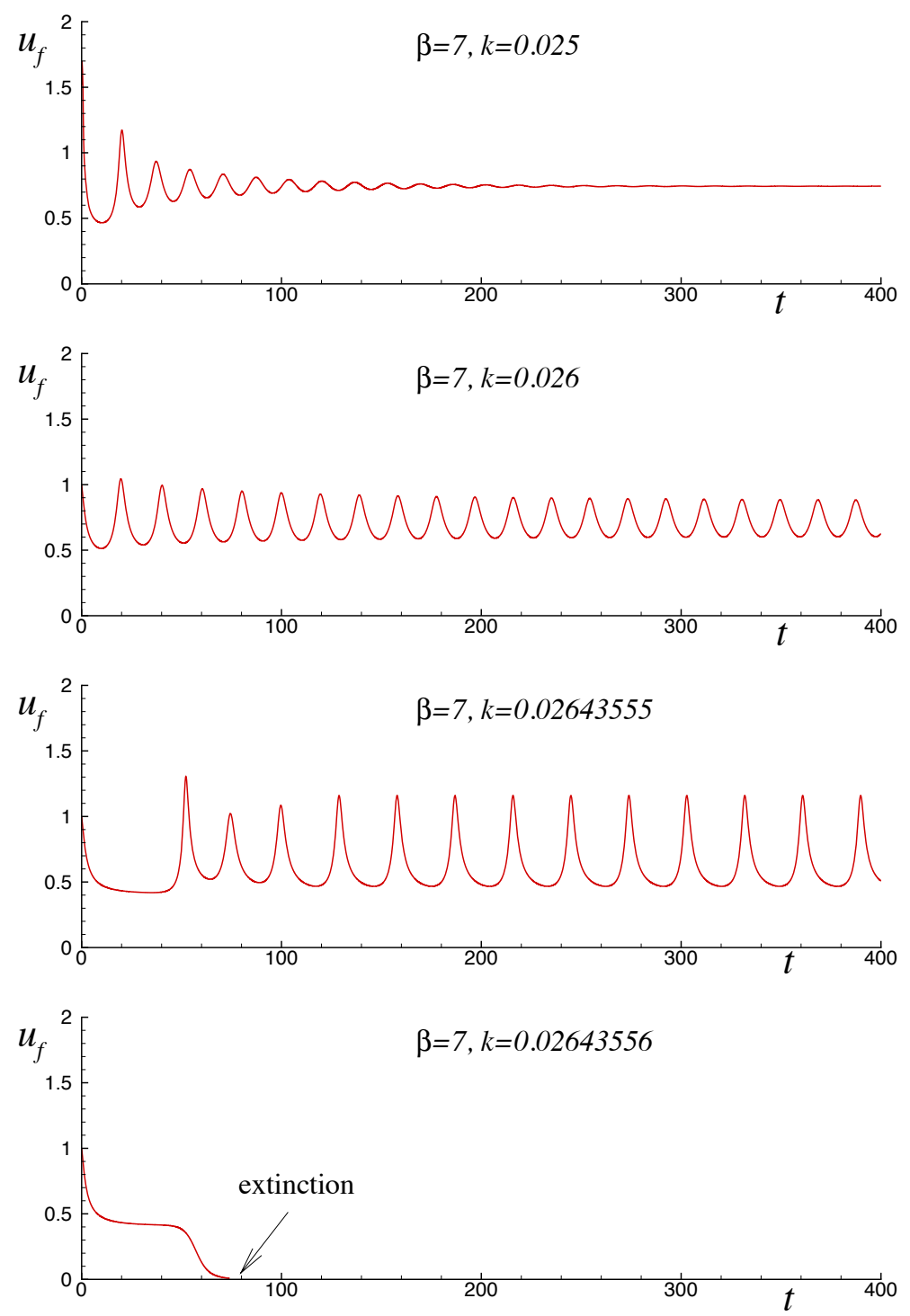

Figure 6: Time histories of the flame velocity calculated for $\beta=7, \gamma=0.7$ and various $k$.

transient stage of behavior a stable steady state while for $k>k_{c}$ the oscillatory dynamics was observed. The growing amplitude of oscillations was detected for increasing values of $k$ leading finally to extinction at $k \approx 0.02643556$. The figure shows that no period doubling bifurcations occur for $\beta=7$ up to the extinction event. It should be noted that the exact value of $k$ corresponding to extinction can be determined only with some uncertainty (estimated below 1\%) because apparently this value depends slightly on initial conditions. One can see in Fig. 1 that 



Figure 7: Examples of time history of the flame velocity calculated for $\beta=8, \gamma=0.7$ and various $k$.

these extinction points (diamond symbols) are significantly lower than both the steady extinction limits given by the turning points (gradient symbols) and the values above which the growth rate $\lambda$ become real (open circles).

For $\beta=8$ the critical value of $k$ above which the steady-state becomes unstable is $k_{c} \approx$ 0.01095 . Figure 7 illustrates the flame dynamics for $\beta=8$ and $k>k_{c}$. One can see that with increasing values of $k$ the flame dynamics changes from merely oscillatory, as shown with the $k=0.015$ case, through the period doubling route illustrated for $k=0.0177$, to the chaotic behavior demonstrated for $k=0.01787$. The solutions with period doubling higher then four are not plotted. The last case shows that the chaotic regime is sustained perpetually without extinction. 

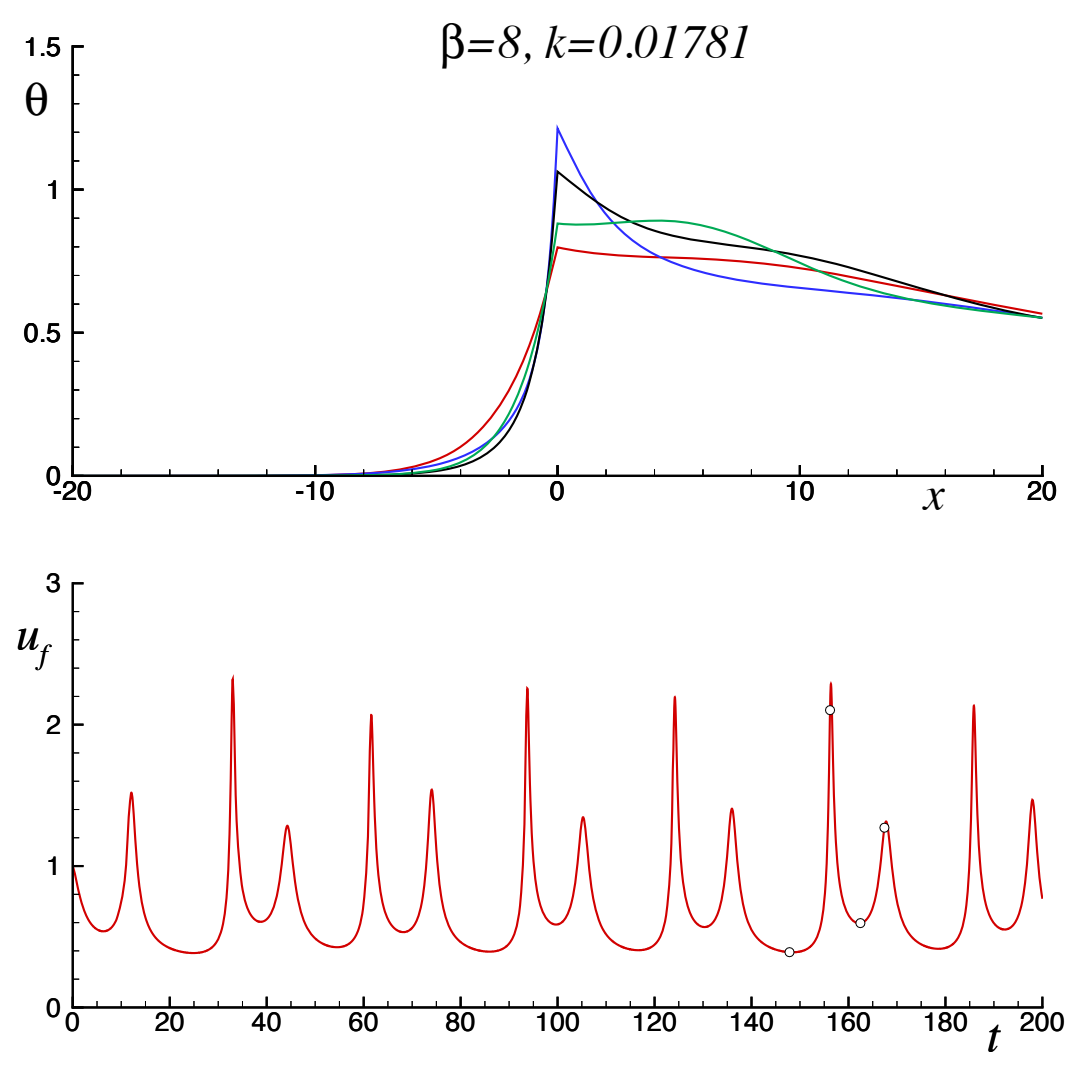

Figure 8: Examples of instantaneous temperature distributions (upper plot) and the time history of the flame velocity (lower plot) calculated for $\beta=8, \gamma=0.7$ and $k=0.01781$; corresponding instants are shown with open circles.

Instantaneous temperature distributions illustrating a typical chaotic dynamics are plotted in Fig. 8 (upper plot) together with the corresponding time-history (lower plot) calculated for $k=0.01781$ where the instants are marked with open circles.

Variations of the flame dynamics are illustrated with the first return map technique. Using the dependence of the flame velocity versus time the series of the local maxima of $u_{f}$ are identified, $\left\{u_{f n}, n=1,2, \ldots\right\}$, where $n$ is the maximum number. The dependence $u_{f(n+1)}$ versus $u_{f n}$ is plotted in Fig. 9 for $\beta=8$ and various $k$. These plots were created using $n>100$ after the starting of simulations, in order to allow the flame dynamics to approach the corresponding attractor.

The numerical simulations indicate that the Feigenbaum's cascade of period doubling bifurcations is produced after the initial PAH bifurcation leading finally to the chaotic regimes 
[26, 27]. The case $k=0.0177$ in Fig. 9 shows the flame dynamics after the first period doubling bifurcation when $u_{f}$ has two different maximums. The second and third period doubling bifurcations are illustrated by plots with $k=0.01777$ and 0.01780 . For $k=0.01781$ the first return map consists of four continuous segments. For $k=0.01785$ the number of continuous segments become equal to two and for $k=0.01787$ the gap between two segments shrinks and the first return map becomes whole continuous. The similar sequence was reported in [24] where the chaotic dynamics was found for the flame propagation in a composite energetic material.

The further increase of heat-losses produces extinction. Figure 10 shows the flame dynamics calculated for three very close values of $k$. One can see that extinction occurs after a long period of a chaotic behavior and the extinction time does not depend on $k$ monotonously. All cases in this figure were calculated starting from the same initial condition given by Eq. (17) with $\theta_{h}=0$ but the extinction times are significantly different despite infinitesimal variations of $k$.

The same sensibility manifests also for very small variations of the initial conditions, as shown in Fig. 11 for $k=0.017875$. One can see that the variations of $\theta_{h}$ of order $10^{-7}$ lead to significant changes in the extinction time. The first return map preceding extinction is shown in Fig. 12. It is interesting that this plot appears as fuzzy in comparison with the return maps in Fig. 9.

This sensibility effect persists also for higher values of $\beta$. Figure 13 shows the time histories of $u_{f}$ calculated for $\beta=9$ and two very close values of $k$. The first case shows the chaotic dynamics without extinction while in the second one the extinction occurs after a long stage of chaotic behavior. One can see that the amplitude of chaotic oscillations grows with $\beta$. The sequence of the first return maps illustrating the chaotic dynamics is shown in Fig. 14. It is interesting that for $k$ close to extinction an additional line indicated with an arrow appears in the first return map plot.

The approximate extinction values of $k$ plotted as a function of $\beta$ in Fig. 4 show high sensitivity of the combustion wave on the heat-loss intensity for large $\beta$. Apparently this dependence approaches zero. It means that even very week heat-losses causes extinction for high values of the Zeldovich number.

\subsection{Intermittency}

Intermittency is well known as the irregular alternation of phases of apparently periodic and chaotic dynamics, see [28]. Intermittent behavior was observed in fluid flows that are turbulent or near the transition to turbulence [29]. This phenomenon was found for the combustion waves 
investigated in the present study. Figure 15 shows the different intervals of the time-history of $u_{f}$ calculated for $\beta=8$ and $k=0.017872$. This case is positioned slightly below extinction. The phases of apparently periodic behavior are indicated with dashed-line circles. It is interesting that intermittency effect was detected only for values of $k$ close to extinction and not at any $k$ for which the chaotic dynamics was observed.

\section{Concluding remarks}

The present study investigates the influence of heat-losses on the combustion wave propagation in narrow samples of energetic materials. For the sake of simplicity, the flame-sheet model is adopted and the heat-loss term is linearized with respect to the temperature field. These simplifications allow to write the steady-state solutions in an analytic form and to carry out, also analytically, the linear stability analysis. It was shown that the increase in heat-losses causes the PAH bifurcation even if the steady-state solution is stable under the corresponding adiabatic conditions. This trend was discovered first in [22] where the role of heat-losses on the stability was investigated for non-adiabatic flame with Lewis number close to unity.

The further increase in heat-losses leads to extinction and two different scenarios were observed. The first one occurs when the Zeldovich number is relatively low. In this case the amplitude of oscillations increases with increasing values of the heat-loss coefficient and the flame extinguishes after reaching the critical amplitude without suffering any period doubling bifurcations. The second scenario was observed when the Zeldovich number is sufficiently high. In this case the Feigenbaum's cascade of period doubling bifurcations is produced after the initial PAH bifurcation leading finally to the chaotic regime. It is found that there exists a range of the heat-loss parameter where the chaotic dynamics can persist infinite time without extinction. But further increase in heat-losses over a limiting value produces extinction. It is interesting that the extinction time can attain a significant magnitude varying also in a stochastic manner near the extinction limit. In reality, this characteristic can be hardly predicted numerically with high accuracy near extinction due to its stochastic nature. This uncertainty is assumed to affect experimental metering of the extinction conditions.

It should be noted that all previous studies of the combustion waves propagation with the chaotic regimes detected were carried out for adiabatic samples. The corresponding Zeldovich numbers chosen as a bifurcation parameter was found very high in these cases. In fact, the chaotic regimes were found in a parametric region corresponding to relaxation oscillations, 
namely where the corresponding linear growth rate of the steady-state perturbations is purely real. This regimes are characterized by very sharp peaks of the flame velocity alternated by extensive intervals with quasi-steady very slow motion. Apparently, including even very small heat-losses in these cases may cause extinction.

In contrast to this, the results presented in the current work show that heat-losses promote the chaotic dynamics which appears for significantly lower values of the Zeldovich number looking much more realistic. It is conceivable that precisely the heat-loss effect becomes the veritable cause of the chaotic behavior observed for flame propagation in narrow solid energetic samples.

\section{Acknowledgments}

VNK acknowledges the support of Spanish MEC under Project \#ENE2015-65852-C2-2-R. VVG acknowledles the support of the Russian Foundation for Basic Research grants \#16-03-00758 and \#17-01-00070.

\section{References}

[1] A.F. Belyaev, L.D. Komkova, Dependence of burning velocity of thermites on pressure, Zh. Fiz. Khim 24 (1950) 1302-1311.

[2] L.B. Maksimov, Issledovanie pulsatsii svecheniya pri gorenii nitroglitserinovih porohov, Zh. Fiz. Khim 37 (1963) 1129-1332.

[3] V.M. Shkiro, G.A. Nersisyan, Structure of fluctuations occurring in the burn- ing of tantalum-carbon mixtures, Combust. Explos. Shock Waves 14 (1978) 121-122.

[4] A.G. Merzhanov, A .K. Filonenko, I.P. Borovinskaya, New phenomena during combustion of condensed systems, Dokl. Akad. Nauk SSSR, Seriya Khimiya 208 (1973) 892-894.

[5] Y.M. Maksimov, A.G. Merzhanov, A.T. Pak, M.N. Kuchkin, Unstable combustion modes of gasless systems, Combust. Explos. Shock Waves 17 (1981) 393-400

[6] T.P. Ivleva, A.G. Merzhanov, Structure and variability of spinning reaction waves in threedimensional excitable media Phys. Rev. E 64 (2001) 036218.

[7] T.P. Ivleva, A.G. Merzhanov, Three-dimensional modes of unsteady solid-flame combustion Chaos 13 (2003) 80-86. 
[8] T.P. Ivleva, A.G. Merzhanov, Three-dimensional unsteady solid flame combustion under nonadiabatic Conditions Combust. Explos. Shock Waves 39 (2003) 300-3008.

[9] V.N. Kurdyumov, C. Jiménez, V.V. Gubernov, A. V. Kolobov, Global stability analysis of gasless flames propagating in a cylindrical sample of energetic material: Influence of radiative heat-losses, Combust. Flame 162 (2015) 1996-2005.

[10] K.G. Shkadinskii, B.I. Khaikin, A.G. Merzhanov, Propagation of a pulsating exothermic reaction front in the condensed phase, Combust. Explos. Shock Waves 7 (1971) 15-22.

[11] M. Gorman, M. El-Hamdi, K.A. Robbins, Chaotic dynamics near the extinction limit of a premixed flame on a porous plug burner, Combust. Sci. and Tech. 98 (1994) 47-56.

[12] V. Gubernov, A. Kolobov, A. Polezhaev, H. Sidhu, G. Mercer, G., Period doubling and chaotic transient in a model of chain-branching combustion wave propagation, Proceedings of the Royal Society of London A: Mathematical, Physical and Engineering Sciences 466 (2010) 2747-2769.

[13] A. L. Sanchez, M. Carretero, P. Clavi, F.A. Williams, One-dimensional overdriven detonations with branched-chain kinetic, Physics of Fluids 13 (2001) 776-792.

[14] B.J. Matkowsky, G.I. Sivashinsky, Propagation of a pulsating reacting front in solid fuel combustion, SIAM J. Appl. Math. 35 (1978) 465-478.

[15] A. Bayliss, B.J. Matkowsky, Two routes to chaos in solid fuel combustion, SIAM J. Appl. Math. 50 (1990) 437-459.

[16] S.B. Margolis, A new route of chaos in gasless combusiton, Combust. Sci. Tech. 88 (1992) 223-246.

[17] I. Brailovsky, G. Sivashinsky, Chaotic dynamics in solid fuel combustion, Physica D 65 (1993) 191-198.

[18] M. Frankel, V. Roytburd, G. Sivashinsky, A sequence of period doublings and chaotic pulsations in a free boundary problem modeling thermal instabilities, SIAM J. Appl. Math. 54 (1994) 1101-1112.

[19] M.L. Frankel, G. Kovačič, V. Roytburd, I. Timofeyev, Finite-dimensional dynamical system modeling thermal instabilities, Physica D 137 (2000) 295-315. 
[20] G. Mercer, R. Weber, H. Sidhu, An oscillatory route to extinction for solid fuel combustion waves due to heat losses, Proceedings of the Royal Society A: Mathematical, Physical and Engineering Sciences, 454 (1998) 2015-2022.

[21] D.A. Schult, Matched asymptotics and the closure problem for combustion waves, SIAM. J. Appl. Math. 60, (1999) 136-155.

[22] G. Joulin, P. Clavin, Linear stability analysis of nonadiabatic flames: diffusional-thermal model, Combust. Flame 35 (1979) 139-153.

[23] J.C. Graña-Otero, Nonlinar dynamics of unsteady premixed, planar flames, Doctoral Thesis, Universidad Politécnica de Madrid, 2009.

[24] V.N. Kurdyumov, V.V. Gubernov, R.V. Fursenko, Controlling of flame propagation in a composite solid energetic material: from stabilization to chaotic regimes, Combust. Flame 182 (2017) 167-178 .

[25] G.I. Sivashinsky, The structure of bunsen flames, J. Chem. Physics, 62 (1975) 638-643.

[26] M.J. Feigenbaum, Quantitative universality for a class of nonlinear transformations, J. Stat. Phys. 19 (1978) 25-52.

[27] P. Coullet, C. Tresser, Iterations dendomorphismes et groupe de renormalisation, J. Phys. Colloques 39 (1978) 25-28.

[28] Y. Pomeau, P. Manneville, Intermittent Transition to Turbulence in Dissipative Dynamical Systems, Commun. Math. Phys. 74 (1980) 189197.

[29] A. D. Staicu, Itermittency in turbulence, University of Technology Eindhoven, 2002. 



Figure 9: The first return maps of the relative maximums of $u_{f}$ plotted for $\beta=8, \gamma=0.7$ and various $k$. 

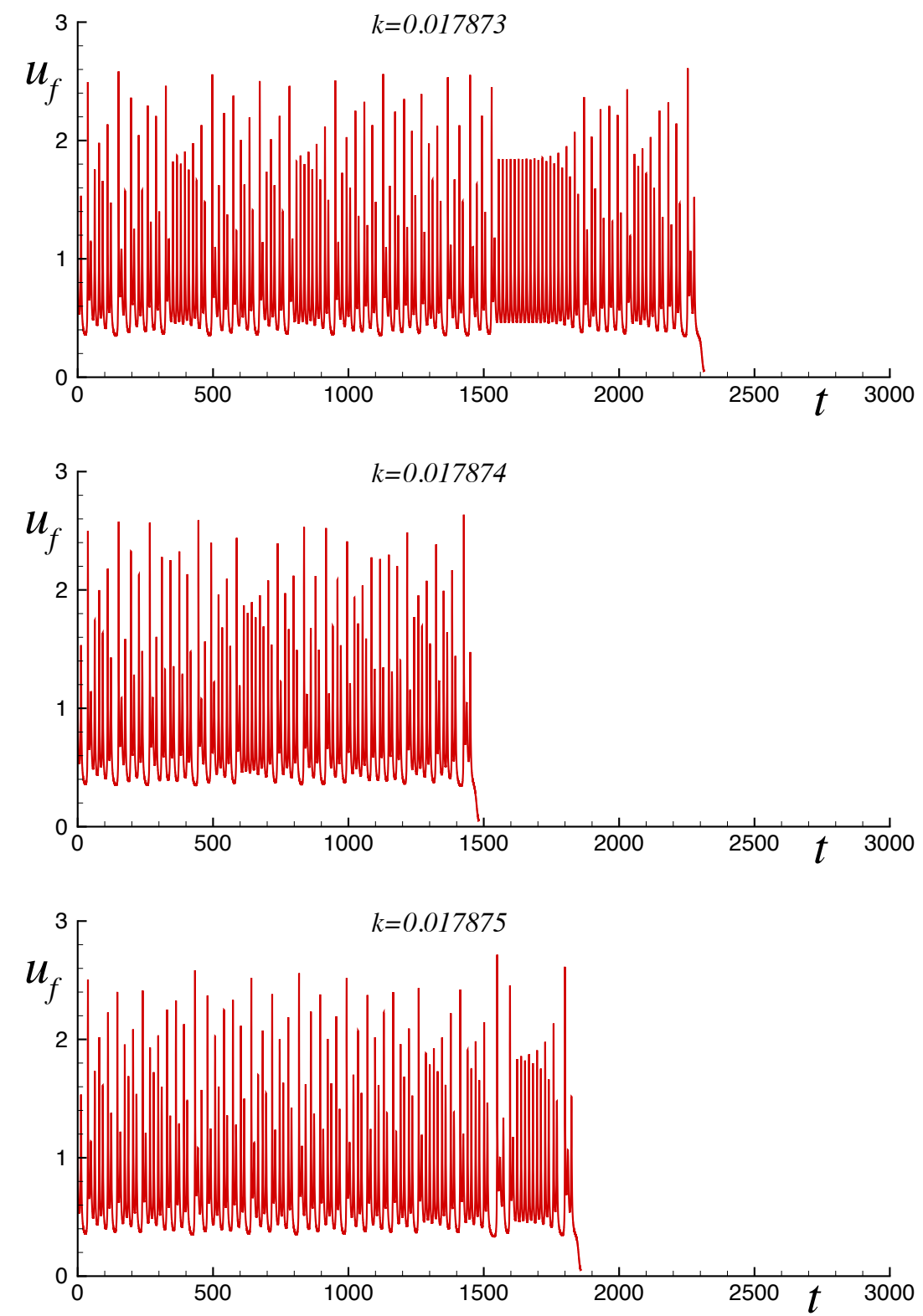

Figure 10: Examples of the chaotic flame dynamics calculated for $\beta=8$ and three very close values of $k$ showing irregular dependence of the extinction time on the heat loss intensity. 

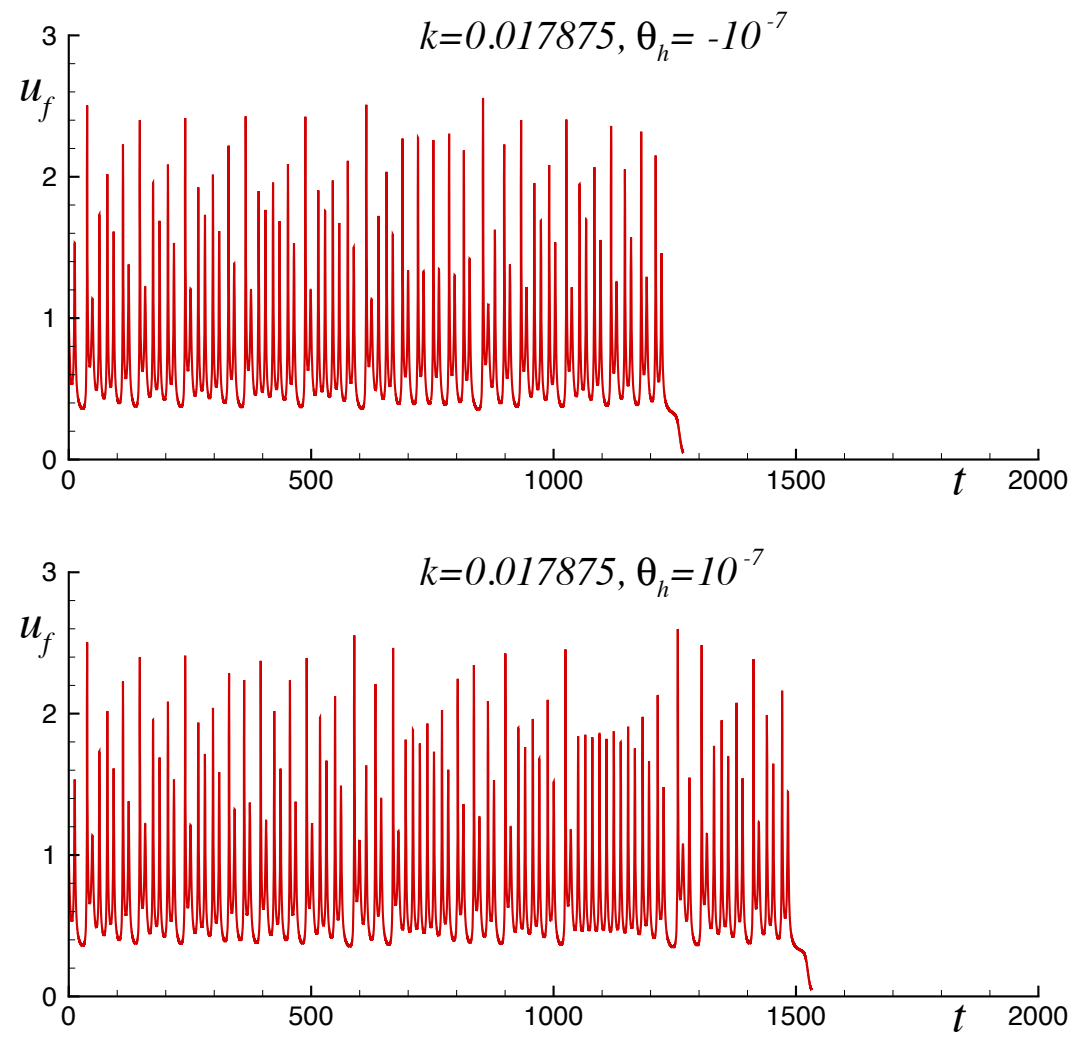

Figure 11: High sensitivity effect of the initial conditions on the extinction time calculated for $\beta=8$ and $k=0.017875$; the case with $\theta_{h}=0$ is shown in Fig. 10. 


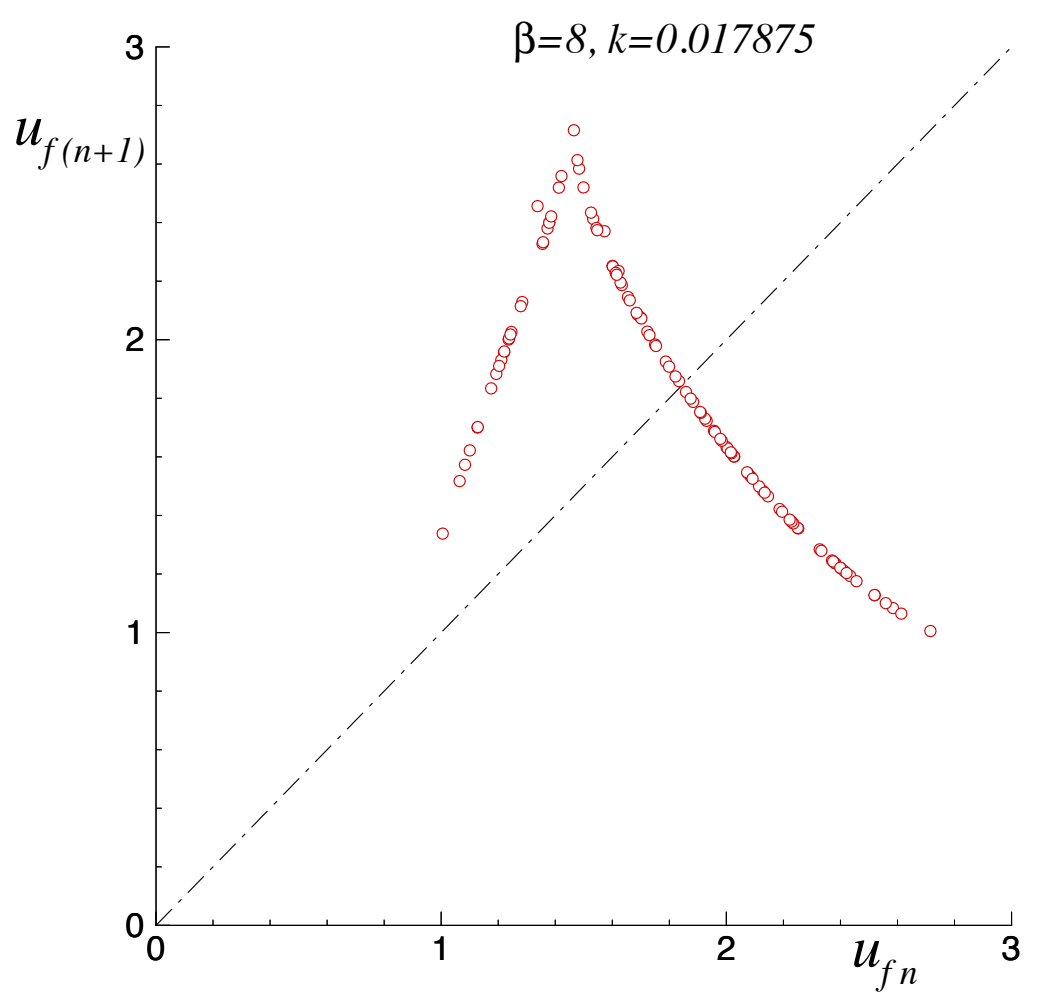

Figure 12: Chaotic first return map preceding extinction, for $\beta=8$ and $k=0.017875$. 

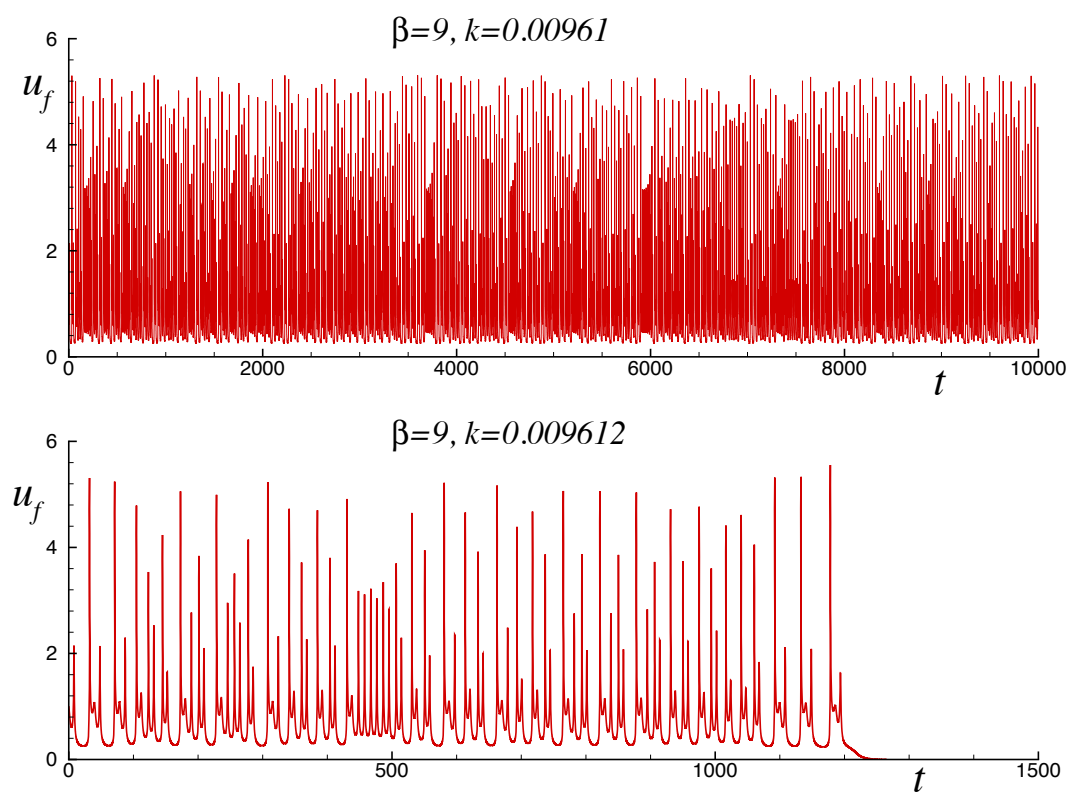

Figure 13: Time histories of the flame velocity versus time calculated for $\beta=9$ and two values of $k$ below and above the extinction value. 

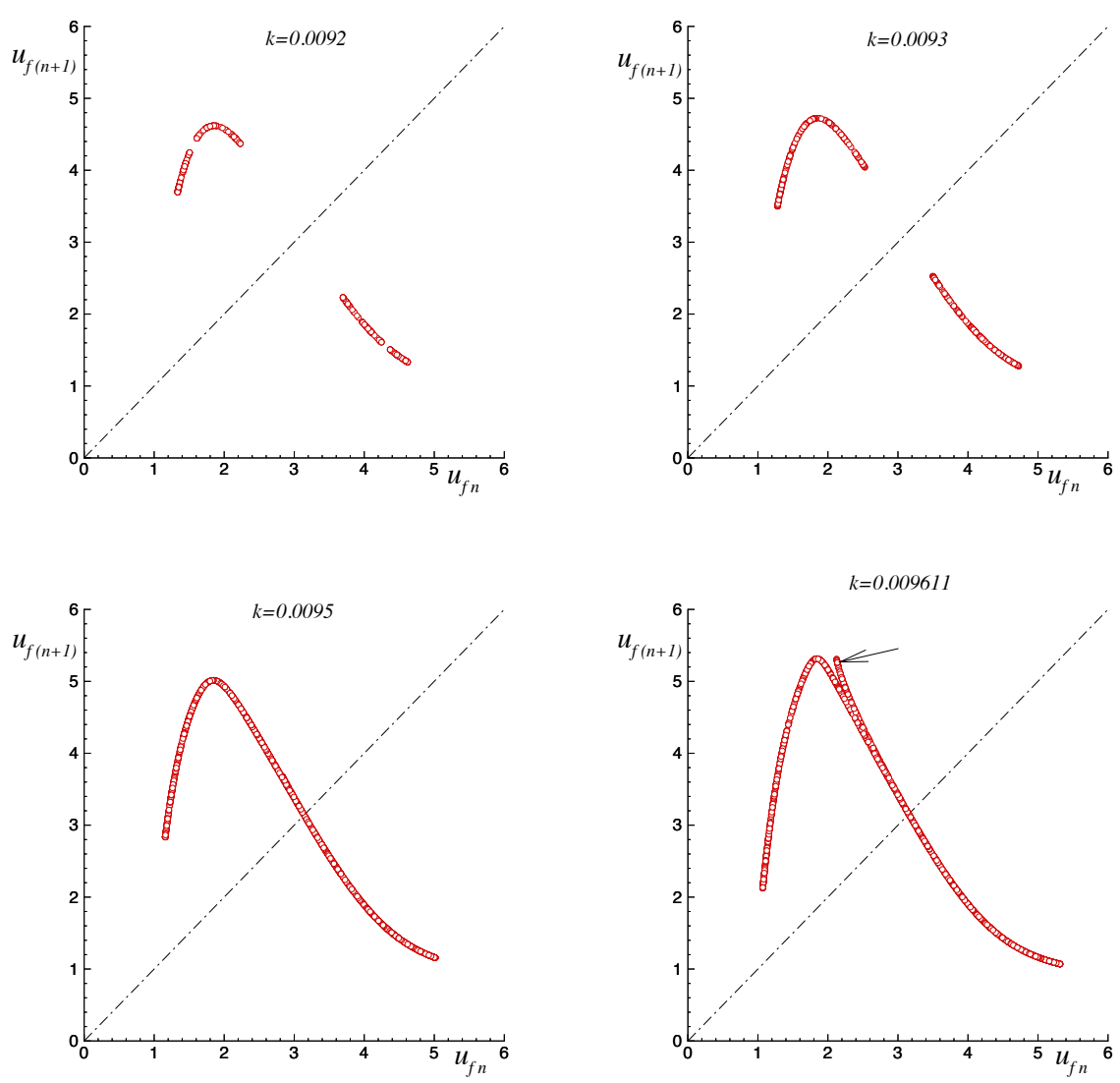

Figure 14: The first return maps calculated for $\beta=9$ and various $k$. 

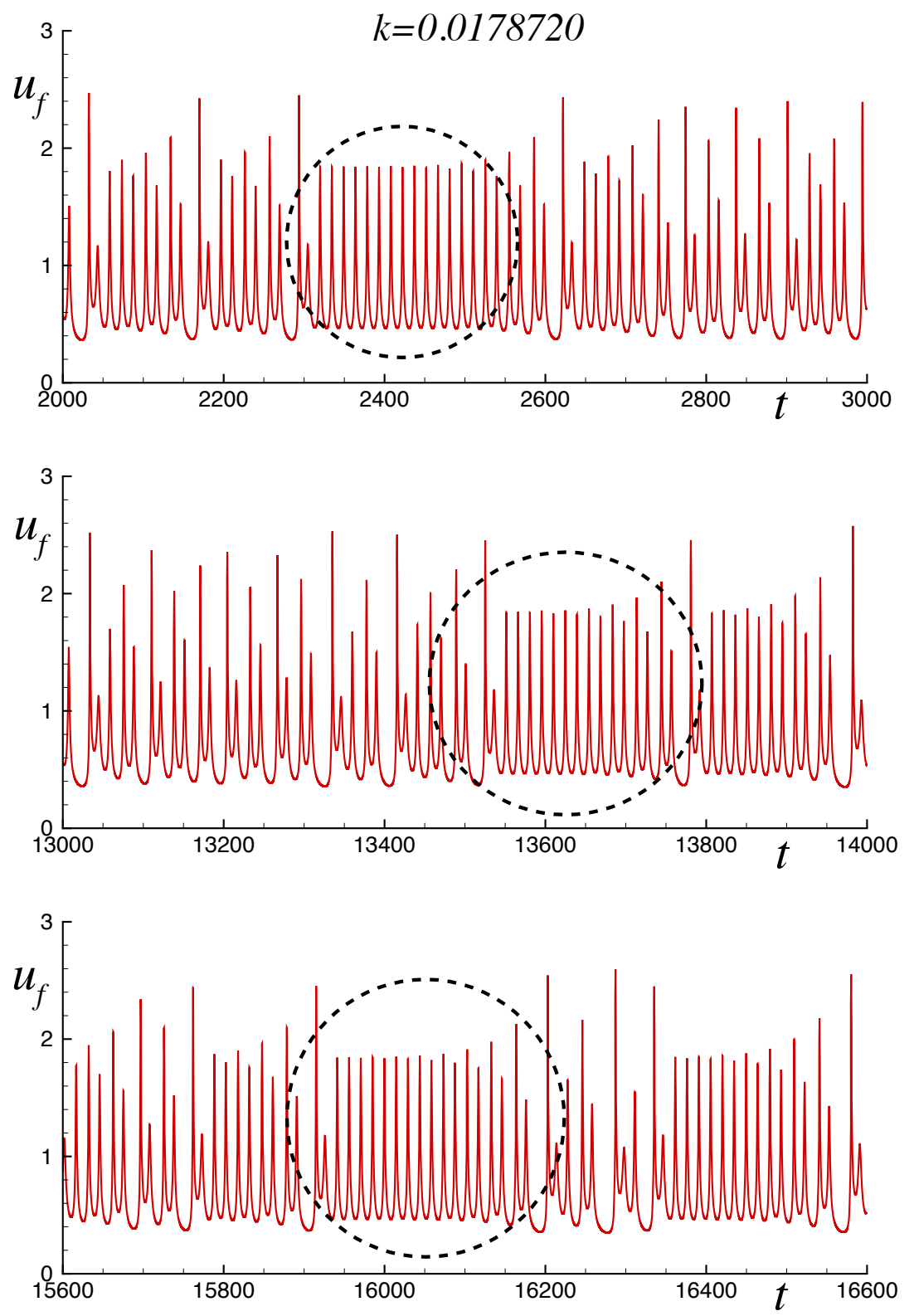

Figure 15: Intermittency effect in the combustion wave: the phases of apparently periodic dynamics are indicated with dash-lines circles, for $\beta=8$ and $k=0.017872$. 